\title{
Concepción Company Company: observando el español desde el pasado
}

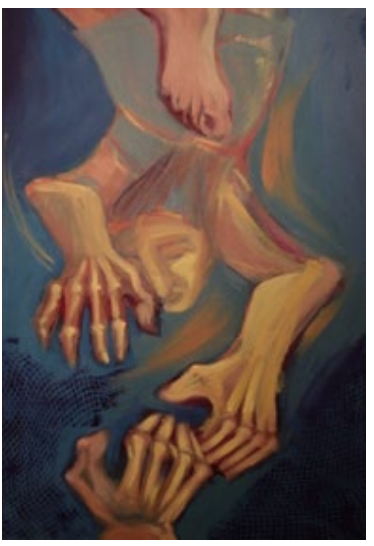

José Luis Ramírez Luengo

Universidad Autónoma de Querétaro, México

joseluis.ramirezluengo@gmail.com

Es muy habitual que, en cualquier conversación que trate sobre la historia del español, termine por aparecer el nombre de Concepción Company Company. Y no es de extrañar, porque la investigadora mexicana ha desarrollado una tarea omnipresente en este ámbito que se plasma en un centenar de investigaciones y varias obras de referencia -entre ellas, sus dos "ojitos derechos": los Documentos Lingüísticos de la Nueva España (1994) y la colectiva Sintaxis Histórica de la Lengua Española (2006, 2009, 2014)-, algo que la ha llevado, entre otros muchos reconocimientos, a ocupar algunas de las más altas responsabilidades académicas en su país, entre las que destacan tres: miembro de número de la Academia Mexicana de la Lengua (2004), investigadora emérita -que no jubilada, como ella misma puntualiza- de la UNAM (2016) y miembro de El Colegio Nacional (2016). En las páginas que siguen la Profa. Company Company dialoga sobre su trayectoria, su visión de la diacronía del español, las preguntas que le quedan aún por responderse y el papel que juega el docente en la formación de las nuevas generaciones de investigadores.

José Luis Ramírez Luengo: Comencemos, si te parece, hablando de tu trayectoria académica; dame una visión general sobre ti misma como investigadora.

Bueno, yo me considero hispanista al cien por cien, y creo que mi toque diferenciador frente al hispanista digamos tradicional es el altísimo interés que tengo en la incorporación de la tipología y de la teoría del cambio lingüístico en la forma de acercarse a los datos de la lengua española. $\mathrm{Me}$ considero filóloga en primer lugar, gramática en segundo e hispanista en general.

\section{En cuanto a tu formación académica...}

Mi formación fue toda en la UNAM: realicé dos años de la licenciatura en la Universidad Complutense, en Madrid, y por diversos azares llegué a México con casi 20 años y me quedé en el país; revalidé algunas materias, pasé el examen de acceso a la UNAM y desde entonces estoy allí, soy producto $100 \%$ UNAM, donde realice la licenciatura, la maestría y el doctorado. Creo que esta formación lleva en sí las características que marcan mi definición como filóloga: mi licenciatura fue en Lengua y Literatura hispánicas, y en 
ella había grandes dosis de literatura, de historia cultural y de lengua, alrededor de un $30 \%$ de cada uno de estos tres troncos disciplinarios. Posteriormente, la maestría y el doctorado fueron en lingüística, pero debido a mi maestro, el argentino Jorge Suárez, y a que así lo permitía el sistema de la UNAM de la época, tomé muchas materias en El Colegio de México y en la Escuela Nacional de Antropología e Historia (ENAH), y eso me permitió abrir horizontes para la lengua española: mi maestro me envió a cursos de totonaco, náhuatl, fonología, lingüística general, tipología, y eso me permitió enmarcar los fenómenos del español, verlo con ojos más abiertos, más incorporados, ver su relación con las lenguas románicas y con las lenguas del mundo... Recuerdo que, cuando llegué a mi primera asesoría con Jorge Suárez, él me dijo "yo no sé nada de español, solo de lenguas oto-mangues y de otras sudamericanas"; "no importa -le dije-, yo le pongo los datos y usted me enseña la teoría", y así comenzamos a trabajar... En esa época, cuando aún no existía internet -mi doctorado fue de 1984 a 1988-, con no poca frecuencia él me decía cosas como "ah, esto también sucede en bereber; para la siguiente reunión tráigame los datos básicos del bereber relacionados con su fenómeno", y entonces yo tenía que salir corriendo para ver dónde se hablaba el bereber, y gracias al Centro de Información de la UNAM -que gratuitamente te proveía del material necesario en una semana- obtenía la bibliografía y los datos para responderle... creo que eso me permitió ver el español con una perspectiva más amplia, insertada en qué es como sistema lingüístico.

Después, mi devenir profesional y personal ha determinado que ahora me defina a mí misma cada vez más como filóloga y menos como gramática, quizá no menos gramática, en realidad, pero sí más en equilibrio entre la gramática interna y la historia externa de la lengua, porque considero que la filología, la literatura y la historia explican fenómenos de la gramática que no se explican por sí solos.

Me resultan muy interesantes estos cambios en la forma de verse uno mismo: yo cada vez me defino menos como hispanista y más como romanista, pues si pones en perspectiva el desarrollo histórico del español, descubres que, en el fondo, todos los sistemas románicos hemos hecho lo mismo, esa idea de que seguimos hablando latín es muy cierta.

Si, tienes razón: si a mí me preguntas, después de 38, de 40 años de trabajo ininterrumpido -mi primer artículo, resumen de mi tesis de licenciatura, apareció en 1983 en la Nueva Revista de Filología Hispánica-, te diría que la esencia de la evolución diacrónica es la continuidad más que el cambio, los parecidos son muchísimos más que las diferencias. La lengua española es prima hermana del portugués, cosa dicha hasta la saciedad, prima cercana del catalán -mal que les pese a tantos- y presenta grandes afinidades con los dialectos del sur de Italia... la continuidad es significativa, y darte cuenta de eso es algo que da el trabajo y la sedimentación de los años investigando.

Ya que hablas de esas variedades, y viendo los lazos históricos del sur de Italia con España, uno pensaría también en influencia por contacto...

Efectivamente, se trata de variedades muy semejantes al español en cuestiones como la falta de transitividad escindida, y que se muestran muy diferentes al toscano, al italiano estándar; seguramente hubo flujos migratorios, lo que no sabemos es la profundidad histórica de tales flujos... En 
este sentido, una idea en la que cada vez me reconozco más es el hecho de que el contacto es la forma natural de vivir de los seres humanos: ya sé que no es decir nada nuevo, pero es que en general los hispanistas nos hemos escandalizado muchas veces con esta cuestión, estamos muy orgullosos de los arabismos, pero no tanto de los galicismos del siglo XIX y mucho menos de los anglicismos actuales. Sin embargo, es precisamente el contacto la forma normal de vivir de los seres humanos, y si el contacto es continuado va a derivar en préstamos, en calcos, en construcciones traspasadas... sí, hay que enfocarse e hincar el diente a los dialectos del sur de Italia, tenemos parecidos en zonas profundas de la sintaxis que no pueden deberse al azar.

Pues habrá que entrar en ese campo, quizá cuando terminemos con América (risas). Yo te quería preguntar ahora sobre tu maestro: ¿cuál fue su gran aporte en tu formación?

Jorge Suárez... en este siglo XXI sería un hombre muy extraño: nunca me habló de tú, siempre de usted... podíamos apostar sobre fútbol, que era su pasión -en mitad de mi doctorado, en 1986, hacíamos apuestas muy extrañas sobre quién ganaría el mundial que se celebraba en México, en parte porque el Estadio Universitario fue sede y el despacho de él estaba en la Torre 2 de Humanidades, a 500 metros, y oíamos los gritos de los asistentes a los partidos-, pero siempre hablándonos de usted. Más allá del anecdotario, como persona tenía dos características: por un lado, una gran generosidad de conocimiento, que probablemente he heredado... imagina, podía llamarme a las once de la noche para decirme que acababa de salir un trabajo en el International Journal of American Linguistics -él fue director un tiempo, y era del consejo de redacción de varias revistas-, y que mañana me lo llevaba para que lo leyera. Esa era una de sus características, y la otra es que, en vez de proporcionar el conocimiento digerido, te hacía preguntas que te echaban a temblar, pues era sumamente exigente, de hecho tuvo pocos discípulos porque no muchos se atrevían a formarse con él; te obligaba a pensar y a tomar decisiones, te decía "esta pregunta tiene dos respuestas buenas, usted me tiene que decir la mejor y por qué lo es".

También le debo agradecer su visión estructuralista: me decía "Concepción, la lengua es forma, y después no hay forma sin significado, sin finalidad comunicativa y sin intencionalidad, pero yo no he visto nunca -recuerdo esa frase, que todavía me repito a mí misma- los significados solos caminando por la calle, pero sí puede uno tocar la forma y probarla, ver sus propiedades y qué nos dice del mundo", y añadía "ustedes los hispanistas están acostumbrados a mirar con ojos subjetivos su lengua porque la hablan, tiene que llegar al punto ideal que es lograr que el español sea para usted alguien lejano a quien opera como cirujano, tiene que ver la lengua como una lengua y no como algo propio que usted habla"; esto es muy importante cuando uno se acerca a datos de lengua, no volcar sobre textos medievales o coloniales opciones del siglo XXI, ni pruebas que yo haría hoy.

Vayamos ahora a tus tareas fundamentales, a tus obras; de todas ellas, ¿cuál te tiene más orgullosa? ¿Cuál es tu ojito derecho?

Pues mi ojito derecho en realidad son dos; si yo echo la vista atrás y me pregunto a qué me he dedicado -esto no lo sabes hasta que no tienes ya la edad que yo tengo-, mis ojos derechos son dos obras de naturaleza diferente pero complementaria: una de ellas es la Sintaxis Histórica de la Lengua Española (SHLE), por supuesto, es la primera de una lengua románica, de gran envergadura, con gran finura de datos y con la teoría totalmente escondida, y esto es importante porque es garantía de que la obra va a seguir siendo consultada, 
pues las teorías pasan, pero los datos bien organizados y descritos permanecen. La SHLE -que en estos momentos no está acabada, tiene ya casi 8000 páginas, con siete enormes volúmenes, y es una obra de referencia que me tiene muy orgullosa- yo la concebí en realidad cuando era estudiante de doctorado: mi tesis trataba sobre el sintagma nominal y en ese momento no había nada, absolutamente nada, sobre la cuestión, porque a mí no me interesaba el sustantivo, lo que me interesaba era el sintagma nominal: qué posibilidades de construcción tiene, qué toma y qué no toma, cómo ha cambiado, si ha cambiado o no... En fin, cuando estaba haciendo mi tesis doctoral yo echaba en falta obras de referencia para el español, todo había que hacerlo y en ese punto picaba piedra -en realidad, seguimos picando piedra con la SHLE: de esta cuarta, y espero que última, parte no se sabe nada...-, yo decía "por qué no habrá una sintaxis histórica de referencia del español como la hay del inglés y del alemán"... los grandes, grandes filólogos son sin duda los ingleses y los alemanes: los ingleses tienen una sintaxis histórica de su lengua desde un punto de vista generativo, una de carácter descriptivo más aséptica teóricamente, otra funcionalista, otra más con una visión combinada de historia externa e interna, y en alemán ocurre lo mismo, hay muchísimo más que en francés, por ejemplo; yo creo que esto probablemente tenga que ver con una tradición protestante, de hermenéutica constante que poseen las sociedades protestantes, donde se tiene que estar constantemente interpretando textos porque gracias a Guttenberg y a Lutero cada uno tiene que tener una Biblia en casa y leerla con ojos propios, creo que eso marca una tradición filológica muy densa en culturas protestantes, y menos densa -sí fuerte, pero no densa- en las de la Contrarreforma. Bueno, ante esta carencia -si tú ves mi libro del año 1992, sorprende la cantidad de bibliografía que hay de otras lenguas, porque ya te digo que no había nada sobre español-, me decía "cuando sea grande, yo quiero hacer una sintaxis histórica".

\section{"Yo quiero hacerme protestante..."}

"Quiero hacerme protestante casi", sí (risas), y entonces en mi primer sabático, tras la estabilidad laboral, quise salir de la UNAM y cambiar de aires, pero por razones familiares -tenía hijos muy pequeños- no podía ni quería irme fuera de México, así que me fui como profesora invitada a El Colegio de México, que tiene una excelente biblioteca; allí no solo di cursos y dirigí dos tesis, sino que me metí a la biblioteca para ver qué había sobre sintaxis histórica del español, creyendo que podía hacer yo sola una obra así, y después de leer y hojear cinco o seis centenas de artículos me di cuenta de que aquello no podía hacerlo yo sola, tenía que ser una obra de otra envergadura, institucional y coral, con investigadores convocados y en la que yo participara como autora, coautora y directora, pues ya sabes que no se trata solo de convocar a los estudiosos, es también darles lineamientos y directrices. En fin, esta SHLE me tiene muy orgullosa, por sí misma, pero también porque sé que la Gramàtica del català antic que están dirigiendo M. Pérez Saldanya y J. Martínez fue convocada a imagen y semejanza de la SHLE, y también sé que en la Universidade Nova de Lisboa y la Universidade de Coimbra siguen la SHLE en la gramática histórica del portugués que están preparando, pues en esta lengua la última es, en realidad, una morfología, la de Nunes...

\section{De los años 30...}

Sí, de los años 30, y también está la de Alí, que es muy buena y tiene más dosis de sintaxis, pero ambas se quedan en la morfología; ahora están haciendo una nueva en esas instituciones, y como te digo también han tomado como modelo esta empresa que emprendimos y sigue viva, y espero que podamos concluir. 
Esta es una de las obras, y mi otro hijo consentido está dentro de la filología: un libro, el primero para América, que son los Documentos Lingüísticos de la Nueva España (DLNE), que apareció en 1994 y que supuso algo de lo que estoy muy orgullosa, que es haber entrado en los archivos. Mira, toda mi formación académica hasta ese momento había sido sobre el castellano medieval: mi tesis doctoral acaba en 1499, la de maestría en el siglo XVI porque quedan ahí residuos de futuros analíticos, la de licenciatura acaba también a inicios del siglo XVI, con los últimos restos de verbos intransitivos puros con ser como auxiliar... en fin, yo estaba muy medieval, y en ese momento apareció la oportunidad de incorporarme al Instituto de Investigaciones Filológicas (IIF) al que pertenezco ahora porque la UNAM, que se estaba quedando sin gente joven, lanzó una convocatoria -a la que yo entré; pertenezco a una generación de primeros becarios de la institución- que permitía estabilizarte si cumplías una serie de requisitos (terminar en tiempo y forma una tesis de calidad adecuada, que obtuviera cum laude y fuera recomendada para libro); yo entré en esa convocatoria y cumplí los requisitos, y entonces J. M. Lope Blanch, que dirigía el Centro de Lingüística Hispánica, me dijo "mire, yo sé que está interesada en incorporarse al Instituto, pero usted debería modernizarse, entienda que a la UNAM el mundo medieval le interesa, pero más aún la historia del español de América". Esa idea de que habría que entrar en los archivos fue suya, aunque él no inició el trabajo filológico, nunca entró a los archivos...

\section{Pero lo propuso...}

Lo propuso, sí, y de hecho él prologó los DLNE... fue un gran dialectólogo, con obras de gran envergadura como el Atlas Lingüístico de México, o las hablas cultas... Entonces me dijo "usted lo que tendría que hacer si quiere concursar como futura investigadora es interesarse en el español americano", y yo les propuse a él y al director del Instituto que haría un corpus documental: pedí una beca en 1988 a la Agencia Española de Cooperación Internacional y a la UNAM para ir a Sevilla, al Archivo General de Indias, luego estuve dos años yendo al Archivo General de la Nación, después al Archivo Histórico de la Ciudad de México... Lo que propuse como metodología -no sabía dónde me estaba metiendo- era buscar documentos cercanos a la proximidad comunicativa, propuse un libro a imagen y semejanza de los Documentos Lingüísticos de Pidal -de hecho, el título, DLNE, sigue totalmente al de Pidal-, un corpus en orden cronológico que abarcara la Nueva España, aunque al final terminó en 300 años de documentos del Altiplano Central, porque Nueva España es un mundo... presenté a inicios de 1993 un corpus de más de mil páginas que se convirtió en el primer libro de una colección, pues ese título se transformó luego en una colección del IIF, y ha dado pie a que se haga filología con ese espíritu de búsqueda de textos que, en la medida de lo posible, reflejen la oralidad de la época. Entonces esta obra me llena de orgullo porque fue mi contacto con el Nuevo Mundo, yo llevaba ya 15 años en México pero el conocimiento profundo de América que creo que tengo, de los marcos culturales, de su evolución gramatical, me lo dio ese libro... yo me hice americana en la filología con este libro.

Posteriormente este libro llevó en una plática que tú conoces con la Dra. Bertolotti en Montevideo, cuando fui a dar un curso, hace muchísimos años: ella, junto con colegas de la Universidad de la República estaban haciendo los documentos para la historia lingüística del Uruguay, de los siglos XVIII y XIX, y les dije "si quieren, cuando ustedes acaben, organizamos un proyecto, convocamos un proyecto", cuya exclusividad es que todos sus documentos están escritos en América y preferentemente por 
americanos. Ese libro de 1994 dio lugar a un corpus digital que es motivo de orgullo para muchos de nosotros, que es el CORDIAM (Corpus Diacrónico y Diatópico del Español de América): la Academia Mexicana de la Lengua lo acogió porque requiere un trabajo de filología y de sistematización, además un convenio con el Instituto Politécnico Nacional generó un motor de búsqueda amigable, y el resultado es un trabajo colectivo, colaborativo, tanto que tú eres coautor de CORDIAM.

En fin, una cosa llevó a la otra, y puedo decir que mi segundo hijo es la filología... yo creo que no haría una gramática histórica con una visión enriquecida desde la teoría, desde hechos de cultura, de historia y de historiografía, si no hubiera entrado en los archivos y me hubiera vuelto filóloga de verdad, es decir, de los que entran en los archivos, eligen los documentos idóneos, ven los problemas ecdóticos, los anotan en una transcripción fidedigna... Entonces estoy orgullosa de esta criatura de 1994, que después dio lugar a otras criaturas y a un corpus electrónico que empieza a ser no pequeño, pues en este momento cuenta con 9017 textos y unos 7.000.000 de palabras.

\section{Y además con transcripciones fiables, que es algo fundamental.}

Muy fiables, porque un requisito, como sabes, es que si el autor no ha entrado al archivo y no ha transcrito los textos, entonces no los recibimos; $\mathrm{y}$ tan fiable queremos que sea que esperamos que en el futuro sea posible acoplar el facsímil con la transcripción, porque no es solo que esté el facsímil, sino que sepas que, si haces una búsqueda, puedes encontrar en los dos, en la transcripción y en el facsímil, el elemento que estás buscando... ese acoplamiento exige un tratamiento informático complejo, pero estamos en vías de lograrlo en pocos años.

Cambiando de tema, ¿̇cuál consideras que es tu gran aporte más allá de las obras concretas, la gran idea que has aportado a la disciplina?

Vamos a ver: si me dices "aporte", yo creo que he hecho obras de infraestructura para la investigación, y así lo manifestó mi director actual, en un proceso de emeritazgo -que no jubilación- concedido por la UNAM en 2016, cuando tuvo que hacer la laudatio de mi perfil. Eso es algo que a veces un teórico desprecia, porque en esas obras de infraestructura no hay hipótesis o marcos teóricos, hay materiales, datos duros y mucha descripción de fenómenos, pero es que eso es lo que queda para las siguientes generaciones: ellos van a tener pavimentado un camino, van a poder hacer mejores trabajos y van a poder llenar vacíos que nosotros hayamos dejado... en esa laudatio el director dijo "Concepción Company es como una pionera" y sí, porque, si piensas, los DLNE fueron pioneros y dieron lugar a otros muchos documentos lingüísticos de otros países hispanoamericanos; si piensas en la SHLE, es también una obra de infraestructura para la investigación... ojalá haya muchos grupos de investigación que digan "vamos a mejorarla, o vamos a hacer otra", cuantas más gramáticas históricas vayamos haciendo, mejor conoceremos nuestra lengua; ojalá haya grupos de gente joven que sean capaces de dialogar y no competir entre ellos, y de poner cada uno su grano de arena para que la protagonista sea la obra: si ves la SHLE, el nombre del autor solo está al inicio de su capítulo y nada más, la protagonista es la obra. Estos son trabajos de infraestructura que estoy segura de que van a quedar para las siguientes generaciones, sea que los usen, sea que digan "hay huecos, hay fallas, y hay que unirse para hacer una obra de mayor envergadura...". 
Y en ese caso también la estarán usando...

También, aunque sea para ver en qué vamos a mejorar, en qué la seguimos, en qué... A este respecto, en la primera parte estaba yo más joven, pues la convocatoria la hice en 2000 y la última parte salió en 2014, no es poco, en 14 años, como te dije antes, hemos sacado casi 8000 páginas, tres grandes partes y ahora está convocada la cuarta... Bueno, en la primera parte yo me puse muy estricta con el número de páginas, y les dije a mis colaboradores -y yo misma me lo impuse en los dos capítulos que escribí- "no debe usted pasar de 100 páginas, y todo lo que le parezca aledaño, marginal, relacionado parcialmente, lo deja fuera"; en la segunda parte me di cuenta de que no iba a ser tan fácil tener otros grupos que hicieran esta empresa en la que la protagonista es la obra y no uno mismo, y entonces me puse mucho menos estricta en cuanto al número de páginas y dije "si usted ve que hay algún fenómeno que está relacionado con su estudio, aunque todavía no sepa cómo, abra una nota, no lo deje fuera", porque desgraciadamente, tal como veo la disciplina de la gramática histórica, que se ha enriquecido mucho -los corpus electrónicos han ayudado a hacer unas generalizaciones que antes serían imposibles-, no soy tan optimista, creo que no estamos dispuestos a colaborar codo con codo, dejando los protagonismos un poco de lado para que el resultado sea la obra misma... entonces he flexibilizado los límites y la concepción de la obra, porque creo que no es tan fácil encontrar grupos que entren al mismo barco y que sepan que tienen que llegar a puerto bien, sin zozobrar; a lo mejor es la edad, que me ha vuelto más flexible, pero creo que lo que hay que hacer es contribuir con datos... creo que ese es mi aporte.

Pero bueno, mi enamoramiento también es la teoría: creo que todos mis trabajos que no son de infraestructura siempre inician planteando un problema teórico, y luego veo cómo aporta y dialoga la lengua española respecto a fenómenos teóricos que son especialmente problemáticos; de hecho, esa es una de las razones por las que en ocasiones escribo, mal que me pese, en inglés, porque la teoría se dialoga en inglés; empieza a dialogarse en español, pero el español no es todavía una lengua de ciencia...

\section{Y eso que no estamos tan mal en comparación con otras...}

No, no, comparado con el portugués, por ejemplo, no digamos el catalán o el italiano, estamos bastante bien, pero estamos lejos de ser una lengua de comunicación científica internacional...

En este sentido, hace tiempo se quejaba el Prof. Veiga, de Santiago de Compostela, de cómo le habían despreciado un artículo porque estaba escrito en gallego; decía que estaba cansado del papanatismo científico que considera que un trabajo, por estar escrito en gallego, es malo o no aporta.

Sí, tiene mucha razón, cuando escribes en una lengua que no es inglés para dialogar... yo estoy convencida de que esto es un problema de cómo ve el mundo teórico lingüístico la trayectoria de las lenguas romances, la ven muy apegada a datos, a anécdotas... Yo estoy convencida -y me ha ocurrido dando clase en EE.UU y en Alemania- de que si llega un investigador con datos de, digamos, el hua, una lengua de Papúa-Nueva Guinea, nada más porque escribe sobre el hua se infiere que va a aportar grandes generalizaciones lingüísticas, tipológicas, teóricas en general, y lo mismo ocurre con las lenguas amerindias. Pero si envías a dictaminar un artículo con datos del español, entonces el dictamen es feroz... en determinadas revistas, los comentarios prácticamente son, si bien te va, "reescriba usted el artículo, 
porque me tiene que decir qué está aportando", y eso aunque esté dicho en la primera página, o directamente te dicen "tiene usted importantes lagunas bibliográficas" porque te faltan dos artículos de 2017 ... o sea, la dictaminación es feroz cuando llegas con artículos sobre español, catalán, gallego, portugués y supongo que otras lenguas románicas, es feroz. Cuando llegas con lenguas denominadas exóticas y que hablan minorías, entonces yo no dudo de que la dictaminación sea dura, pero hay otra mirada, mucho más benévola... Con las lenguas romances hay que batallar doble, pero como no vamos a cambiar la mentalidad de las culturas que escriben en la lengua científica internacional, que son culturas de imperio, dominantes, entonces lo que hay que hacer es seguir batallando y trabajar...

Pues seguiremos, qué vamos a hacer... Y ahora que digo lo que tenemos que hacer, ¿qué pregunta tienes en la cabeza para responder en el futuro? ¿qué cuestión general te causa ruido actualmente...?

Bueno, hay un hecho concreto que son los fenómenos de discurso, cómo se estructura el discurso y el párrafo, pero están vinculados al esqueleto de la cuarta parte de la SHLE, no es una pregunta teórica, en realidad son preguntas concretas: cuáles han sido los grandes cambios en la estructura argumental de la lengua española, en la estructuración del párrafo... en general, los grandes cambios asociados al discurso y a la estructura argumental e informativa, porque una cosa es "el niño come peras", que es una posibilidad de la lengua española, pero en los datos reales de lengua eso no lo dice nadie. Yo estoy ahora enfrascada en el complemento circunstancial, que tiene numerosas restricciones y debe ir después del verbo, como en "los niños estudian en la escuela"... pues bien, ese "en la escuela" pospuesto, de un corpus de más de 4200 ejemplos entre 1250 y el siglo XXI, es menos del $1 \%$. Pero eso son curiosidades; lo que está detrás es cuál es el acoplamiento entre el uso real de la lengua y lo que se propone para ella, eso es una pregunta que me causa curiosidad, y cada vez que avanzamos en una parte de la SHLE esa curiosidad va quedando subsanada, pero me doy cuenta de que el abismo entre lengua en uso y teoría es tremendo.

Ahora bien, hay un punto -en el que no soy nada pionera- que me interesa enormemente, una cuestión que ya se preguntaron Menéndez Pidal, Lapesa, Cuervo... los grandes grandes historiadores de la lengua, y por eso te decía antes que cada vez soy más filóloga. Ellos ya se lo preguntaron, aunque quizá no se respondieron del todo, pero ahí está la pregunta: se trata de cuál es la relación entre la lingüística histórica interna, los cambios que parecen dinámicas internas, y la historia externa de la lengua. Tras 40 años de trabajo en gramática histórica, tengo la firme convicción de que la última palabra de los cambios no está en la lengua, está en la historia, en la cultura, en la literatura, en las modas sociolingüísticas y comunicativas... entonces me produce una gran inquietud saber cuál es el balance entre gramática histórica interna y externa: creo que no son disciplinas separadas, como nos han explicado siempre, y tengo verdadera curiosidad por saber cuál es el balance y las complementaciones, cuáles son las dosis que necesitas de historia externa para explicar fenómenos de gramática interna, porque con esta puedes explicar qué pasó y cómo se produjo un cambio, pero no puedes explicar el porqué. El porqué último -tú, que eres filólogo nato, seguro que piensas igual- no está en el sistema, está en la cabeza de los hablantes, en las estructuras sociales, en si la lengua en cuestión está al amparo de estructuras gubernamentales que la acogen y que fomentan una cultura escrita y oral en ella, o por el contrario está desamparada del poder, alejada de los marcos sociales y culturales hegemónicos... eso me 
causa gran, gran curiosidad. No creo que sea una quinta parte de la SHLE porque creo que no viviré para verlo, pero esa es mi pregunta, el porqué... considero que mis trabajos explican bien el qué y el cómo pasó algo, pero los porqués... esos -creo yo- solo están en la historia, en lo que arropa a la lengua y a su empleo.

Esto nos lleva a la interdisciplinariedad, que está ahora tan de moda: parece que uno tiene que incorporar el concepto a los proyectos porque vende y queda bien, pero lo cierto es que es desde ahí desde donde se explican muchas cosas...

Efectivamente, en el fondo es así: creo que las disciplinas de cambio, sea variación sincrónica, tipología, gramática histórica o las que trabajan adquisición y hechos de neurolingüística, son las únicas que pueden hacerse esa pregunta última del porqué, y creo también que esta interdisciplinariedad es una realidad, por eso digo que soy cada vez más filóloga: mira, yo he incursionado en algunos siglos que me son especialmente gratos, el siglo XIX es la centuria del discurso, cuando la lengua española se discursiviza como ahora, y el siglo XVIII -que me interesó especialmente- es cuando la gramática, no el discurso, se vuelve casi casi como la actual; pero hay un siglo que es todo un reto, que es el XVII, porque cuando aplicas estadística, cuando haces diacronías profundas, este siglo hace quiebre constantemente... siempre digo que la SHLE sin el siglo XVII hubiera tenido muchas menos páginas, y menos costo en lo económico y en lo moral (risas).

\section{Pero sería mucho menos interesante...}

Mucho menos interesante. Ese siglo es un misterio. ¿¿ué ocurrió en ese siglo? Probablemente lo que ocurrió se encuentra en la historia externa, que explica la manipulación -manipulación, recreación, creación...- del código, es algo exhuberante; yo, cuando aplico un modelo estadístico, ya sé que va a hacer quiebre el siglo XVII, es más, la sorpresa es que no lo haga: si ves toda la SHLE, ese siglo siempre hace quiebres, y estamos lejos de saber por qué, de ese porqué que solo pueden contestar las disciplinas que atañen a fenómenos de cambio y que se interesan por un diálogo que está en el mundo interdisciplinario. Por eso yo cada vez soy más filóloga, porque eso es lo que hacían nuestros maestros, ¿no? Si te fijas en Pidal, yo diría que Pidal es un historiador antes que un lingüista, un gran historiador... uno no puede irse de esta vida sin leer La España del Cid (1929), por ejemplo, o sus Orígenes del español (1926); las dosis que tiene de filología, de cultura, de datos históricos para explicar pequeños fenómenos de fonología o de morfología... Quizá es que estoy envejeciendo, pero cada vez soy más filóloga y menos lingüista (risas). Aunque me siguen enamorando las hipótesis... como dicen por ahí, "es mejor una mala hipótesis que ninguna hipótesis", porque por lo menos una mala hipótesis te guía unas búsquedas, sea para entrar en un archivo, sea para ordenar unos datos, sea para explicar un fenómeno, pero la ausencia de hipótesis te hace dar palos de ciego...

Bueno, en realidad no son cuestiones incompatibles: "tengo la teoría; ahora voy a observar desde otro punto de vista, con los datos externos...".

Así es, cada vez la historia externa me produce más, más curiosidad, y de hecho me veo en mi escritorio cada vez con más libros de historia: antes compraba libros de gramática, lingüística, teoría... y últimamente me doy cuenta de que he leído mucha historia, mucho de vida cotidiana, de historia conceptual, de la relación estado-iglesia -clave para muchos periodos-, de la relación entre estructuras de poder y estamentos bajos... En fin, cada 
vez me doy más cuenta de que voy a una librería y me compro libros que tienen que ver con estas áreas. O con la literatura, que siempre he usado en mis estudios, pero que ahora leo con unos ojos culturales que antes no tenía, antes leía literatura con ojos de gramática...

\section{Buscando la estructura...}

Buscando la estructura, pero ahora leo literatura buscando el marco cultural en que aquella obra fue producida... Ya te digo, no sé si es la edad o los muchos años de estar trabajando con los datos y ver la imposibilidad de encontrar el porqué, que no está en el dato...

Vamos a ir terminando ya, una última pregunta: ¿qué quieres inculcar a tus discípulos? ¿qué consideras que es fundamental enseñarles? A mí mi maestra, Carmen Isasi, me enseñó que lo fundamental es la honradez científica y la prudencia al tratar los datos. En tu caso, ¿qué quieres transmitirles como cuestión de principio?

Como cuestión básica me oigo una y otra vez señalar lo mismo: el compromiso. Es decir, que uno no puede dejar las cosas a medio hacer, que tiene que dar lo mejor de sí mismo en cada trabajo que haga, no abandonar, y ese compromiso requiere una base científica muy sólida... una vez comienzas algo, hay gente que está confiando en ti y tú estás confiando en ti mismo, y por eso debes cumplir. Quizá es esto mismo que te decía Carmen: el compromiso implica que no puedes fallarte a ti mismo haciendo chafadas... luego traduces el mexicanismo chafadas.

\section{Chapuzas.}

Chapuzas. No puede ser. Obviamente, no quiere decir que, después de 35 años, si yo trabajara los tiempos compuestos no lo haría de otra forma, por supuesto que sí: otra experiencia, otros corpus, otros ojos... Pero en aquel momento hice lo mejor que pude. A los jóvenes les digo que un trabajo que está bien hecho hay que atreverse a publicarlo, hay que tener la humildad de aceptar la crítica, y aceptar que la crítica es constructiva, y que tiene que venir y no pasa nada. No dejar la investigación en un cajón es ya un compromiso, tanto con la lengua española como con las instituciones que nos pagan... yo creo que es un privilegio trabajar en lo que te gusta, ser pagado -y bien pagado- por hacer lo que te gusta, y esas oportunidades de vida y de trabajo hay que aprovecharlos. Así que estas son las cuestiones fundamentales: compromiso, seguridad, atreverse a la crítica y tener rigor, ser absolutamente explícito y honesto en los pasos que estás dando, y que haya un respaldo científico en todo lo que desarrollas, no andar haciendo trampeos ni chapuzas. Eso es lo que espero que aprendan, la actitud y el compromiso.

Perfecto. No sé si quieres añadir algo más, para que no aparezcan solamente mis preguntas...

(Duda). Sí... ¿qué espero de mí misma cuando tengo esta edad, que son 63 ? Primero, espero tener la lucidez de saber cuándo tengo que retirarme aunque no tenga la obligación, es decir, tener la lucidez mental de saber que quizá lo que estoy diciendo ya no aporta... ojalá la tenga. Y en cuanto a mis discípulos, me siento muy afortunada porque me busca mucha gente joven brillante, comprometida; me gustaría que, cuando yo tenga ya 80 años, digan "esta señora me enseñó una actitud ante la disciplina, me enseñó a ver fenómenos de lengua con ojos abiertos". Quizá cuando uno pasa los 60 comienza a preguntarse cómo se quiere ver dentro de 20 años; eso es lo que quisiera: comprender que debo retirarme si ya no tengo la apertura 
de ojos ni la flexibilidad suficiente para cuestionarme cosas, si no tengo preguntas que hacerme o nada nuevo que buscar en los textos. Uno tiene que estar orgulloso de lo que hizo en el pasado, no tiene que arrepentirse porque fueron diferentes etapas de su vida, pero tampoco puede dormirse en la complacencia, más en nuestros países, que hacen enormes esfuerzos económicos por financiar la investigación; es poco correcto, y por eso tengo que tener la lucidez de saber cuándo me debo retirar. 